\title{
Biomarker-based patient selection improves stroke rehabilitation trial efficiency
}

Cathy M. Stinear, $\mathrm{PhD}^{1}$

Winston D. Byblow, PhD²

P. Alan Barber, MBChB, PhD, FRACP ${ }^{1,3}$

Suzanne J. Ackerley, $\mathrm{PhD}^{1}$

Marie-Claire Smith, $\mathrm{PhD}^{1}$

Steven C. Cramer, $\mathrm{MD}^{4}$

1. Department of Medicine, Centre for Brain Research, University of Auckland, Auckland, New Zealand

2. Department of Exercise Sciences, Centre for Brain Research, University of Auckland, Auckland, New Zealand

3. Neurology, Auckland District Health Board, Auckland, New Zealand

4. Department of Neurology, University of California Irvine, California, USA

Corresponding author

Cathy Stinear, PhD, Department of Medicine, University of Auckland, Private Bag 92019, Auckland 1142, New Zealand (c.stinear@auckland.ac.nz)

@StrokeScience (Stinear)

@MyPlasticBrain (Byblow)

@DrMCSmith (Smith)

Keywords: stroke; rehabilitation; neurophysiology; biomarker; transcranial magnetic stimulation

Subject Areas: ischemic stroke; intracranial hemorrhage; rehabilitation 


\section{Abstract \\ Background and Purpose}

Inter-subject variability complicates trials of novel stroke rehabilitation therapies, particularly in the sub-acute phase after stroke. We tested whether selecting patients using motor evoked potential (MEP) status, a physiological biomarker of motor system function, could improve trial efficiency.

\section{Methods}

A retrospective analysis of data from 207 patients (103 women, mean (SD) 70.6 (15.1) years) was used to estimate sample sizes and recruitment rates required to detect a 7-point difference between hypothetical control and treatment groups in upper-limb Fugl-Meyer and Action Research Arm Test scores at 90 days poststroke. Analyses were carried out for the full sample and for subsets defined by motor evoked potential (MEP) status.

\section{Results}

Selecting patients according to MEP status reduced the required sample size by $75 \%$ compared to an unselected sample. The estimated time needed to recruit the required sample was also reduced by $72 \%$ for patients with MEPs, and was increased by 2-3-fold for patients without MEPs.

\section{Conclusions}

Using biomarkers to select patients can improve stroke rehabilitation trial efficiency by reducing the sample size and recruitment time needed to detect a clinically meaningful effect of the tested intervention. 


\section{I ntroduction}

The acute treatment of ischemic stroke has been transformed by trials that select patients for reperfusion therapies based on advanced imaging biomarkers. ${ }^{1}$ Rehabilitation research could also adopt an approach that uses biomarkers to select patients with the greatest potential to benefit from a putative therapy. ${ }^{2}$ However, stroke is a heterogeneous condition with high interindividual variability in recovery. ${ }^{3}$ This reduces statistical power in rehabilitation trials, particularly during the sub-acute stage. ${ }^{2}$ A potential biomarker for hand and arm motor recovery is the presence of motor evoked potentials (MEPs) determined using transcranial magnetic stimulation (TMS). Patients with MEPS have a functionally intact motor cortex and lateral corticospinal tract, and better motor recovery. ${ }^{4}$ We determined whether trial efficiency could be improved by using MEP status for patient selection.

\section{Materials and Methods}

The largest available dataset of patients with acute stroke and known baseline MEP status ${ }^{5}$ was used to estimate the sample sizes required to detect rehabilitation benefits on upper-limb motor performance at 90 days after stroke. Inclusion criteria were age $\geq 18$ years and monohemispheric ischemic stroke or intracerebral hemorrhage with new upper-limb weakness within the previous 72 hours. Exclusion criteria were contraindications to TMS, cerebellar stroke, communication or cognitive impairment precluding informed consent, and residing out of area precluding follow-up. Only $2 \%$ of screened patients were contraindicated for TMS. ${ }^{5}$ All participants provided written informed consent and completed usual care.

Baseline clinical assessments were made and MEP status of the paretic upperlimb determined within 7 days post-stroke. Participants were considered MEP+ if an MEP of any amplitude and consistent latency ( $\pm 3 \mathrm{~ms}$ ) could be elicited in the paretic extensor carpi radialis or first dorsal interosseous muscles on half of at least eight consecutive trials, with the target muscles either at rest or during attempted voluntary activation. ${ }^{5}$ Upper-limb motor impairment was evaluated with the upper-limb Fugl-Meyer scale (UE-FM, maximum=66). Upper-limb motor function was evaluated with the Action Research Arm Test (ARAT, maximum $=57$ ). These scales are valid, reliable, and recommended by international consensus; ${ }^{6}$ scores were obtained 90 days after stroke.

Analyses were carried out for the full sample, and repeated for the MEP+ and MEP- subsets. Population estimates of UE-FM and ARAT scores 90 days poststroke were obtained using bootstrapping with replacement, 10,000 samples, and bias corrected accelerated estimates of $95 \%$ confidence intervals. These estimates were then used to calculate the sample sizes required to detect treatment effects of 7 points on the UE-FM and ARAT scores 90 days after stroke, assuming two-tailed tests, alpha $=0.05$, and $1: 1$ randomization, with statistical power of 0.80 and 0.90 . A 7-point treatment effect was chosen 
because it is approximately $10 \%$ of each scale, and there are currently no agreed upon minimal clinically important differences for these scales at the subacute stage of stroke.

\section{Results}

In all, 207 patients (103 women, mean (SD) 70.6 (15.1) years) completed baseline and 90-day follow-up assessments (Table 1). The full sample and MEP+ subset $(n=177)$ had similar demographic and baseline clinical characteristics. The MEP- subset $(n=30)$ had more severe stroke and greater upper limb impairment at baseline. As expected, MEP+ patients had higher estimated population mean 90-day UE-FM and ARAT scores than MEP- patients. The estimated percentage of patients who are MEP+ was $85.5 \%(95 \% \mathrm{Cl} 81.2 \%$ $89.9 \%$ ) giving a relative recruitment rate of 0.855 for MEP+ and 0.145 for MEP-, compared to recruiting patients regardless of MEP status.

For both UE-FM and ARAT, the estimated required sample size to detect a treatment effect at 90 days for MEP+ patients was only 27 - 29\% compared to the full sample of unselected patients (Table 2 ). Recruiting the required sample size for MEP+ patients would thus take approximately one third of the time needed to recruit unselected patients. The estimated required samples size to detect a treatment effect at 90 days for MEP- patients was 31\% for UE-FM, and $20 \%$ for ARAT, relative to the full sample (Table 2). However, recruiting the required sample size for MEP- patients would take approximately three times longer for the UE-FM, and almost twice as long for ARAT, than for unselected patients.

\section{Discussion}

MEP status is a biologically relevant and non-invasive biomarker of corticospinal tract function that clinicians can obtain at the bedside after minimal training. ${ }^{5}$ Selecting patients on the basis of MEP+ status reduces variance in outcome measures at 90 days, and is estimated to reduce sample size and recruitment time by around two-thirds, without limiting the pool of participants. Selecting patients on the basis of MEP- status also reduces variance and the required sample size by at least two-thirds. Corticospinal tract biomarkers might also be relevant for broader outcomes, such as independence, and warrant further investigation along with neuroimaging and blood biomarkers.

This small retrospective study reflects the characteristics of patients who may be suitable for participation in trials of upper-limb rehabilitation interventions. It serves as an example of how biomarkers could be used to enrich samples for stroke rehabilitation trials. Patients, clinicians, investigators, and funding agencies stand to gain from improved trial efficiency. Using biomarkers for patient selection in stroke rehabilitation trials could markedly increase trial sensitivity and efficiency, with associated decreases in participant burden, research costs, and time required for completion. 


\section{Acknowledgements}

We thank Matthew Petoe PhD for assistance with data acquisition, and Sharon Yeatts PhD for advice on statistical analyses.

\section{Sources of Funding}

Health Research Council of New Zealand (09/164 and 11/270, Byblow, Stinear, Barber).

\section{Disclosures}

None

\section{References}

1. Mokin M, Rojas H, Levy El. Randomized trials of endovascular therapy for stroke--impact on stroke care. Nat Rev Neurol. 2016; 12:86-94

2. Stinear CM. Prediction of motor recovery after stroke: Advances in biomarkers. Lancet Neurol. 2017; 16:826-836

3. Prabhakaran S, Zarahn E, Riley C, Speizer A, Chong JY, Lazar RM, et al. Inter-individual variability in the capacity for motor recovery after ischemic stroke. Neurorehabil Neural Repair. 2008;22:64-71

4. Byblow WD, Stinear CM, Barber PA, Petoe MA, Ackerley SJ . Proportional recovery after stroke depends on corticomotor integrity. Ann Neurol. 2015; 78: 848-859

5. Stinear CM, Byblow WD, Ackerley SJ, Smith MC, Borges VM, Barber PA. PREP2: A biomarker-based algorithm for predicting upper limb function after stroke. Annals of clinical and translational neurology. 2017;4:811820

6. Kwakkel G, Lannin NA, Borschmann K, English C, Ali M, Churilov L, et al. Standardized measurement of sensorimotor recovery in stroke trials: Consensus-based core recommendations from the stroke recovery and rehabilitation roundtable. Int J Stroke. 2017; 12:451-461 
Table 1. Baseline clinical and demographic characteristics

\begin{tabular}{|c|c|c|c|}
\hline & Full Sample & MEP+ & MEP- \\
\hline & $N=207$ & $\mathrm{~N}=177$ & $\mathrm{~N}=30$ \\
\hline Age $(y)$, mean (range) & $70.6(18-98)$ & $71.5(18-98)$ & $65.3(32-90)$ \\
\hline Sex, female (\%) & $103(49.8)$ & $84(47.5)$ & $19(61.2)$ \\
\hline \multicolumn{4}{|l|}{ Ethnicity, n (\%) } \\
\hline European & $131(63.3)$ & $117(66.1)$ & $14(46.7)$ \\
\hline Māori & $10(4.8)$ & $9(5.1)$ & $1(3.3)$ \\
\hline Pacific & $30(14.5)$ & $21(11.9)$ & $9(30.0)$ \\
\hline Asian & $36(17.4)$ & $30(16.9)$ & $6(20.0)$ \\
\hline \multicolumn{4}{|l|}{ Stroke risk factors, $\mathrm{n}(\%)$} \\
\hline Hypertension & $133(64.5)$ & $112(63.3)$ & $21(70.0)$ \\
\hline Dyslipidemia & $66(31.9)$ & $53(29.9)$ & $13(43.3)$ \\
\hline Previous cardiac disease & $56(27.1)$ & $52(29.4)$ & $4(13.3)$ \\
\hline Atrial fibrillation & $47(22.7)$ & $41(23.2)$ & $6(20.0)$ \\
\hline Diabetes mellitus & $43(20.8)$ & $35(19.8)$ & $8(26.7)$ \\
\hline Ex-smoker & $35(16.9)$ & $31(17.5)$ & $4(13.3)$ \\
\hline Smoker & $17(8.2)$ & $12(6.8)$ & $5(16.7)$ \\
\hline First stroke, n (\%) & $181(87.4)$ & $155(87.6)$ & $26(86.7)$ \\
\hline \multicolumn{4}{|l|}{ Stroke type, n (\%) } \\
\hline $\mathrm{TACl}^{\mathrm{a}}$ & $12(5.8)$ & $6(3.4)$ & $6(20.0)$ \\
\hline $\mathrm{PACl}^{\mathrm{b}}$ & $74(35.7)$ & $59(33.3)$ & $15(50.0)$ \\
\hline $\mathrm{LACl}^{\mathrm{c}}$ & $84(40.6)$ & $78(44.1)$ & $6(20.0)$ \\
\hline $\mathrm{POCl}^{\mathrm{d}}$ & $16(7.7)$ & $14(7.9)$ & $2(6.7)$ \\
\hline $\mathrm{ICH}^{\mathrm{e}}$ & $21(10.1)$ & $20(11.3)$ & $1(3.3)$ \\
\hline Hemisphere, left, n (\%) & $99(47.8)$ & $93(52.5)$ & $6(20.0)$ \\
\hline Hand, dominant, n (\%) & $95(45.9)$ & $88(49.7)$ & $7(23.3)$ \\
\hline Intravenous thrombolysis, n (\%) & $19(9.2)$ & $17(9.6)$ & $2(6.7)$ \\
\hline Endovascular thrombectomy, n (\%) & $3(1.4)$ & $1(0.6)$ & $2(6.7)$ \\
\hline NIHSS ${ }^{f}$ score, median (range) & $4(0-19)$ & $4(0-16)$ & $11(2-19)$ \\
\hline NIHSS score $0-4, \mathrm{n}(\%)$ & $112(54.1)$ & $111(62.7)$ & $1(3.3)$ \\
\hline NIHSS score $5-15, \mathrm{n}(\%)$ & $85(41.1)$ & $62(35.0)$ & $23(76.7)$ \\
\hline NIHSS score $\geq 16, \mathrm{n}(\%)$ & $10(4.8)$ & $4(2.3)$ & $6(20.0)$ \\
\hline \multicolumn{4}{|l|}{ UE-FMg score } \\
\hline Mean (SD) & $41.6(20.8)$ & $47.2(16.9)$ & $8.5(3.8)$ \\
\hline Range & $2-65$ & $2-65$ & $4-21$ \\
\hline
\end{tabular}

a. Total anterior circulation infarct.

b. Partial anterior circulation infarct.

c. Lacunar infarct.

d. Posterior circulation infarct, excluding cerebellar stroke.

e. Intracerebral hemorrhage.

f. National Institutes of Health Stroke Scale.

g. Upper-extremity Fugl-Meyer scale, maximum $=66$, higher scores indicate less motor impairment. 
Table 2. Estimates of 90 day scores, required sample sizes, and relative time to recruit, with $95 \%$ confidence intervals

\begin{tabular}{|c|c|c|c|}
\hline & $\begin{array}{l}\text { Full Sample } \\
\mathrm{N}=207\end{array}$ & $\begin{array}{l}\text { MEP+ } \\
\mathrm{N}=177\end{array}$ & $\begin{array}{l}\text { MEP- } \\
\mathrm{N}=30\end{array}$ \\
\hline \multicolumn{4}{|l|}{ UE-FMa score at 90 days } \\
\hline Estimated mean ${ }^{\mathrm{b}}$ & $52.2(49.8-54.6)$ & $58.2(56.8-59.5)$ & $17.1(13.5-21.2)$ \\
\hline Estimated SD ${ }^{b}$ & $17.1(15.0-18.9)$ & $8.7(7.3-10.1)$ & $11.3(8.2-13.6)$ \\
\hline \multicolumn{4}{|l|}{ Sample size ${ }^{c}$} \\
\hline Statistical power $=0.80$ & $190(148-232)$ & $52(38-68)$ & $82(46-122)$ \\
\hline Statistical power $=0.90$ & $254(196-319)$ & $68(48-90)$ & $112(60-162)$ \\
\hline \multicolumn{4}{|l|}{ Relative time to recruit $^{d}$} \\
\hline Statistical power $=0.80$ & & $0.32(0.30-0.34)$ & $2.98(2.14-3.63)$ \\
\hline Statistical power $=0.90$ & & $0.31(0.29-0.33)$ & $3.04(2.11-3.50)$ \\
\hline \multicolumn{4}{|l|}{ ARAT $^{\mathrm{e}}$ score at 90 days } \\
\hline Estimated mean ${ }^{\mathrm{b}}$ & $43.6(41.2-46.0)$ & $49.8(48.3-51.2)$ & $7.2(4.3-10.4)$ \\
\hline Estimated SD & $17.7(15.7-19.4)$ & $9.5(7.9-11.0)$ & $8.8(6.8-10.1)$ \\
\hline \multicolumn{4}{|l|}{ Sample size ${ }^{c}$} \\
\hline Statistical power $=0.80$ & $204(160-244)$ & $60(42-80)$ & $52(32-68)$ \\
\hline Statistical power $=0.90$ & $272(214-316)$ & $80(56-106)$ & $70(42-90)$ \\
\hline \multicolumn{4}{|l|}{ Relative time to recruit ${ }^{d}$} \\
\hline Statistical power $=0.80$ & & $0.34(0.31-0.38)$ & $1.76(1.34-1.92)$ \\
\hline Statistical power $=0.90$ & & $0.34(0.31-0.39)$ & $1.78(1.35-1.96)$ \\
\hline
\end{tabular}

a. Upper-extremity Fugl-Meyer scale, $\max =66$, higher scores indicate less motor impairment.

b. Estimates of population means and standard deviations (SD) for scores 90 days after stroke, obtained using bootstrapping.

c. Required sample size to detect a 7 point difference in scores between hypothetical treatment and control groups at 90 days after stroke.

d. Time to recruit the required sample size of MEP+ or MEP- patients, relative to the time required to recruit the required sample size of unselected patients.

e. Action Research Arm Test, $\max =57$, higher scores indicate better motor performance. 